\title{
Taldea: A Tool for Fostering Spontaneous Communities
}

\author{
Ghada Ben Nejma ${ }^{1}$, Philippe Roose ${ }^{1}$, Jérôme Gensel ${ }^{2}$, \\ Marc Dalmau ${ }^{1}$, and Mohamed Amine Ghorbali ${ }^{2}$ \\ ${ }^{1}$ LIUPPA Laboratory / T2I Research Team \\ 2 Allée du Parc de Montaury 64600 Anglet, France \\ ${ }^{2}$ LIG Laboratory / STEAMER Research Team \\ 681 rue de la Passerelle, BP72 \\ 38402 Saint Martin d'Hères cedex, France \\ \{gbennej, roose, dalmau\} @iutbayonne.univ-pau.fr, \\ \{Jerome.Gensel, mohamed-amine.ghorbali\}@imag.fr
}

\begin{abstract}
This paper presents the design and development of a community application that facilitates user's access to communities and exchange information between community members. The application provides multiple services in order to satisfy the needs of users sharing a common interest or practice. We introduce a new type of ephemeral communities with geolocalization, that we call spontaneous communities. Our approach is based on an ontology, which models the different components of a community and their relations. The proposed approach has been validated through a prototype for the visitors of a botanical garden.
\end{abstract}

\section{$1 \quad$ Introduction}

Recent years have witnessed rapid advances in the technological infrastructure. The democratization of mobile devices (e.g. smartphones, touchpads, laptops, sensors, etc.) has made information accessible to anyone at anytime and from anywhere. The idea of ubiquitous computing or computers everywhere has been first proposed by Mark Weiser [8]. In heterogeneous and highly changing environment, ubiquitous applications are progressing to the detriment of distributed systems. Today, ubiquitous applications include more and more the community notion (e.g. Yuback ${ }^{1}$, Foursquare $^{2}$ ). The growth of communities is justified by:

- $\quad$ Social information needs (e.g information for citizenship);

- Social interaction and communion needs (e.g. discussions and sharing of experiences);

- $\quad$ Social recognition needs (e.g political leader recognition).

- Etc.

Yet, social information is not fully exploited as underlined by Deparis et al. [1]: "the organizations begin to realize that they lose a part of their knowledge by not

\footnotetext{
${ }^{1}$ http: / / www . yuback. com/

${ }^{2}$ https : / / fr. foursquare.com/
} 
capitalizing social fragments. Actual knowledge management tools are not able to correctly handle these social fragments and their value is lost for the organizations". Thus, organizations use more and more community applications to facilitate social interaction between users and capitalize the exchanges.

In the MOANO ${ }^{3}$ (Models and Tools for Territory Discovery-oriented Nomadic Applications) Project of the ANR (French National Research Agency), we are interested in the design and the development of a spatiotemporal context-sensitive community application in order to facilitate the discovery of a territory. The objective of this work is to propose a community model and to present a tool for fostering spontaneous communities. The ontology-based community modeling allows us to organize and represent the different components of a community and their relationships, to make information search easier for users, to infer new knowledge and capitalize social data. This paper presents our approach for modeling communities using semantic web technologies (e.g. $\mathrm{FOAF}^{4}, \mathrm{SIOC}^{5}$ ). The use of these technologies offers a wide range of benefits such as interoperability, data portability and semantics data interpretation.

The paper is organized as follows. The first section compares the various social tools that can be used by organizations. In the second section, we propose a usage scenario of our community application. It describes how the visitor of a botanical garden can join or create communities in a specific geographic area. In section 3, we describe functionalaspects of our community application. After presenting different modules allowing users access to communities and exchange information, concluding remarks summarize the importance of the presented approach and outline some future work.

\section{Social Networks versus Community Applications}

The popularity of socials networks and online communities has grown exponentially "Social networks offer to users interesting means and ways to connect, communicate, and share information with other members within their platforms" [4]. They are more and more used by organizations to facilitate collaboration between users groups and other aspects of information exchange. Several studies suggest methods for discovering communities in social networks. They consist in identifying groups of users more interconnected than the rest of the network. A comparative analysis of community discovery methods in social networks is addressed by the paper of Sathik et al. [5].

The acquisition, collection and analysis of information spread over social networks are difficult tasks for organizations. Furthermore, with the growing number of social networks, it is often hard to motivate users to join the same network. Some solutions have been proposed for organizations such as enterprise social networking to help employees share their expertise and connect with others, especially in geographically distributed organizations. However, organizations have to face a high number of new challenges such as mobility, ubiquity, and problems of heterogeneity. For all these

\footnotetext{
${ }^{3}$ http: //moano. liuppa.univ-pau.fr/

${ }^{4}$ http: //www. foaf-project.org/

${ }^{5}$ http://sioc-project.org/
} 
reasons, we consider that context-aware community applications are a solution to adapt to changing situations. We have made a comparative study between social networks, enterprise social networks and community applications and we present the results in the table 1.

The existing applications (e.g. eklaireur ${ }^{6}$ ) offer services and information related to a specific theme, which has to be defined in advance by developers. The creation of new communities with other themes cannot be spontaneous by user. In our communities' applications, we define a new type of communities as "a spontaneous group of individuals having a common interest related to a circumstantial, accidental, incidental or fortuitous situation that occurs somewhere on a geographical territory". This kind of community can meet specific needs, which are generally not taken into account by perennial communities (e.g. accidents, natural disaster, crisis, fire, etc.).

Table 1. Comparison Table of different social tools in Organizations

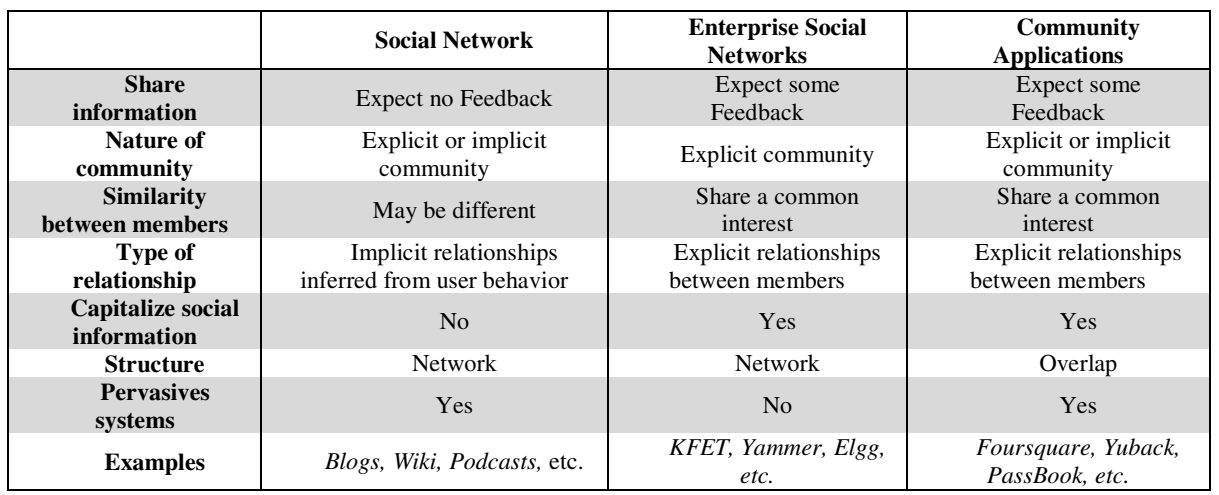

\section{Use Case}

Our work focuses more particularly on temporary, short-lived communities. Indeed, it is the type of community that best matches with unexpected situations. The proposed application intends to help the user in the creation of a spontaneous community with the objective of answering an incidental situation. However, our community application, called Taldea, is not only an application that assists user to create a community, but also consists of an environment that supports communities throughout their life cycle. We briefly describe a scenario in a botanical park Mosaïque, located near the French city of Lille. In this park can be found a variety of stakeholders (visitors, gardeners, eco-guards, external participants...). These stakeholders can be equipped with mobile devices. Taldea is deployed thanks to a service-based reconfiguration platform we have developed, named Kalimucho. This platform implements a contextual-deployment heuristic in order to find a configuration that matches the current context with QoS requirements. Taldea is composed of interconnected components supervised by the platform. In this paper, we

6 https://play.google.com/store/apps/details?id=com.eklaireur.ek ldroidshl=fr 
choose to detail the different ways to access a community. We have implemented the following scenario. A fan of orchid flowers visits the park Mosaïque to discover the local flora of the region. Equipped with his Smartphone, she enters the park. The Smartphone automatically integrates Taldea through Geofencing ${ }^{7}$. Communities that are semantically related to orchid flowers will be recommended to the visitor. Moreover, she can search for a community by formulating a query. For instance, let us suppose that the visitor connects to the community of interest 'fans of orchids'. During her tour in the botanical garden, she identifies some rare 'Ophrys apifera' flowers. She first takes pictures of her discovery. Then, she consults the list of connected members of the "fans of orchids" community. However, at this moment, no member is present in the park or is available for a real time communication. Then, she decides to publish a topic (the unit of exchange within a community) in the community space "fans of orchids" to invite people to admire these flowers. While waiting for an answer from the members, the user of Taldea decides to create a spontaneous community named "fans of rare orchids". Taldea classifies this new community as a sub-community of the "fans of orchids" community. The visitor then creates the topic "Discover Ophrys apifera". This topic is annotated with spatiotemporal data in order to facilitate the location of flowers. The created topic is recommended to all the users of Taldea (they can be members of other communities) presents in the park. A short time later, she received several answers for her topic. Several interested persons moved on the scene to discover or to admire these flowers. Other scenarios can be foreseen for other communities such as the communities of practice (e.g. the gardeners of the park). Critical situations (e.g. accidents, disasters, etc.) can highlight the importance of spontaneous communities. In the next section, we describe the different ways to access communities.

\section{Taldea: Access Mechanisms to Communities}

As shown in the following example, there are three ways to access a community in Taldea: recommendation, search and creation of a community (cf. figure 1). (1) The user logs in, (2) her interests are mapped to the botanical ontology, (3) as well as the interests of existing communities. Then, similarity measures are used in order to identify communities semantically similar to the user's profile. If the user is not satisfied with the proposals (4), Taldea, allows her to enter a query using natural language for searching for other communities. (5\&6) The query is annotated through the web service TextAnnot ${ }^{8}$.(7) Sparql query is formulated from annotation results to query the ontology by the Reasoner.(8) If the user is not satisfied with the search results, she can create a spontaneous community.(9) Inference rules that can be used

7 The geo-fencing approach is based on the observation that users move in a virtual perimeter for a real-world geographic area. It allow us to track people on a mobile subscriber list based on proximity to a particular retail store and sending them tailored messages relating to that store.

8 A web service developed by our research team, it provides information in the form of some annotations based on botanic ontology (http://themat2i.univ-pau.fr:8080/ TextAnnot-WWW/annotation. j.sp). 
to infer new knowledge for further enrichment of community description. (10) Finally, the community ontology is instantiated with the user- entered information and the inferred information.

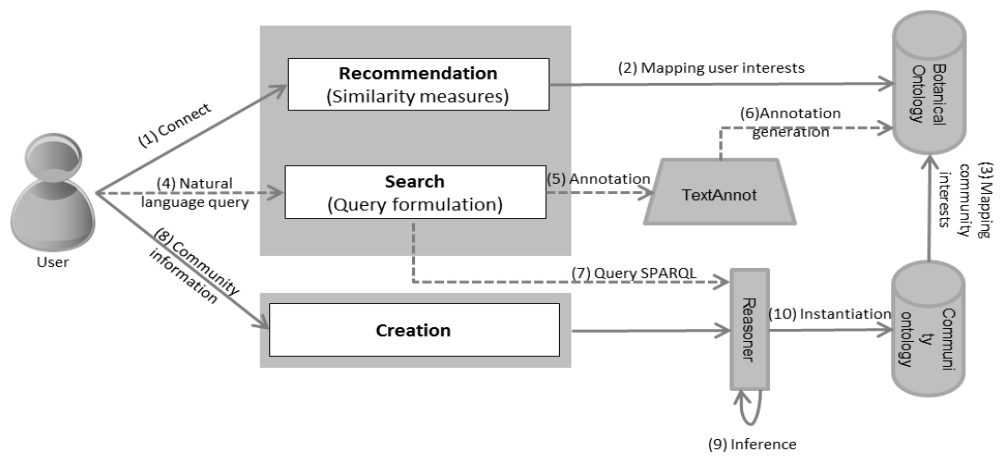

Fig. 1. Access mechanisms to communities

\subsection{Creation and Closing of the Community}

Nowadays, the social fragments [1] become essential to enrich the knowledge base of organizations. Several ongoing projects aim to use some social information produced by communities such as the projects SPIPOLL ${ }^{9}$, operation Escargot ${ }^{10}$, Sauvage de ma rue $^{11}$. Referring to the same ontology for different communities (all users refer to the same vocabulary) can enrich the knowledge base of the organization. New knowledge can be reused by other communities.

In this section, we present the ontology for describing formally a community using multiple standards such as FOAF, SIOC, Owl-Time ${ }^{12}$ and GeoRSS ${ }^{13}$, etc. These standards make it possible for software agents to understand information exchanged without the ambiguity, complex processing, and rigidity brought by other representation formalisms (e.g. natural language, relational database). Figure 2 shows the community ontology (partial definition of the ontology). The model is structured around a set of abstract entities, each describing physical or conceptual objects including Interest, Member, Lifespan, Resource, Location, Type.

Each creation of a community is an instantiation of the ontology concepts (cf. figure 2). Additional knowledge is provided from user's input annotation and the inferences rules, inferences engines (i.e Reasoner) such as the type of community. As illustrated in Table 2, the user enters the following description of the community "fans of rare orchids". This description is automatically annotated. TextAnnot attach the created community to the concept 'Orchid' of the botanic ontology.

\footnotetext{
${ }^{9}$ http: //www.spipoll.org/

10 http: / / www . noeconservation . org/index 2 php? rub=12\&srub=31\&ssrub= $322 \&$ goto $=$ contenu

${ }^{11}$ http: //sauvagesdemarue.mnhn. fr/sauvages-de-ma-rue/presentation

${ }^{12}$ http: / / www .w3 . org/TR/owl-time/

${ }^{13}$ http: //georss.org/Main_Page
} 


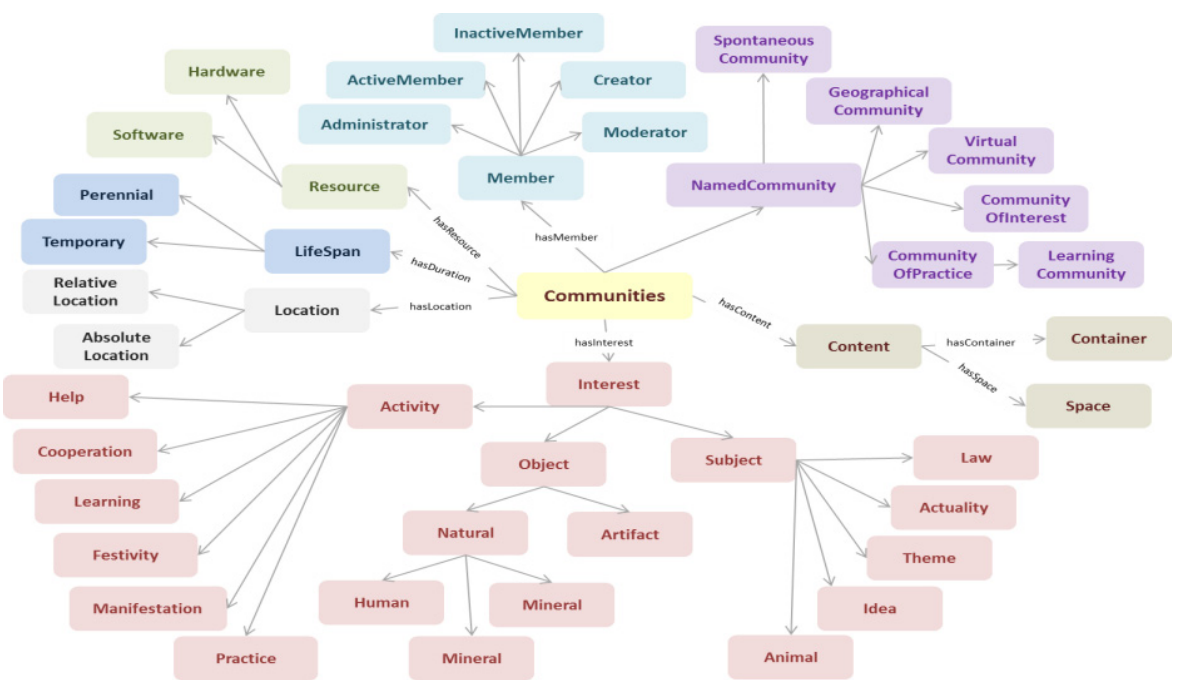

Fig. 2. A part of the community ontology

Table 2. An example of inference

\begin{tabular}{|l|}
\hline Terminology box associated with the community ontology (Tbox) \\
\hline Communities: \{CommunityOfInterest, CommunityOfPractice, GeographicalCommunity, \\
SpontaneousCommunity, VirtualCommunity\} \\
Interest $\{$ Activity, Object, Subject\} \\
Object $\{$ Artifact, Natural\} \\
Natural \{Animal, Human, Plant, Mineral\} \\
CommunityOfInterest $\subseteq$ Communities $\cap \exists$ hasInterest (Object $\vee$ Subject) \\
\hline Assertion box associated with the community ontology(Abox) \\
\hline Community $\cap$ hasInterest (Orchid) \\
Orchid: Plant \\
\hline Inference \\
\hline Fans of rare orchids $\rightarrow$ CommunityOfInterest \\
\hline
\end{tabular}

A community may be closed when the need for which it was created becomes satisfied or obsolete. After closing a community, the extracted knowledge exchanges between members are capitalized.

\subsection{Access to Communities}

\subsubsection{Community Recommendation}

In this paper, we propose a method of community recommendation based on a measure of semantic similarity between the interests of the user and those of communities. 


\subsubsection{User's profile}

There are different profile representations in different contexts such as CC/PP ${ }^{14}$ (Composite Capability/Preference Profiles), PAPI ${ }^{15}$ (Public And Private Information), FOAF (Friend of a friend), etc. All these profile representations are widespread standard. We have chosen the FOAF vocabulary to represent the user's profile because it is simple and enables interoperability among systems. Moreover, given that it is based on RDF, the vocabulary can be extended as needed. Information, in FOAF profile, is categorized as FOAF basics, Personal info, Online accounts, Projects and groups, Documents and image. In community recommendation, we are particularly interested in the class Interest to perform some semantic matching between the user's profile and communities.

\subsubsection{Measure of Similarity between the User's Profile and the Community}

In the field of information retrieval, semantic similarity measures are used to assess the semantic proximity between the query and the document. In the context of community applications, we evaluate the semantic similarity between the user's profile and communities, by the mapping of user interests and community interests in the domain ontology (i.e the botanic ontology). In different contexts, several approaches have been proposed to measure semantic similarity between concepts in ontology. We can distinguish three major approaches: The first type is based only on the hierarchy or the edge distances, the second type is based on the nodes and the third type is the hybrid approach [6]. For measuring semantic similarity between user profile and communities, we adopted an approach based on arcs because other approaches used the frequency information. This is not significant in the case of community because it is created around infrequent concepts in the corpus. We choose the measure of $\mathrm{Wu}$ and Palmer [9]. It is simple to implement, has good performance compared to other similarity measures [6].

The Measure of Wu and Palmer [9] is a measure between concepts in an ontology. Wu and Palmer, similarity metric measures the depth of the two concepts in the ontology, and the depth of the least common subsumer (LCS), and combines these figures into a similarity score:

$$
\operatorname{sim}(\text { concept } 1, \text { concept } 2)=\frac{2 * \operatorname{depth}(L C S)}{\operatorname{depth}(\operatorname{concept} \mathbf{1})+\operatorname{depth}(\operatorname{concept} \mathbf{2})}
$$

\subsubsection{Community Recommendation Algorithm}

We propose an approach to recommend communities to users based on their interests. Alg. 1 shows the details of our approach. The key idea is to compute semantic similarity between the user's profile and communities. We first project the user's interests and communities' interests on the domain ontology (Line 1). Secondly, we compare the interests and return communities whose interests match user's ones (Line 2 5). If no match is found, we compute the semantic similarity between each user's interest and each community's interest using Wu and Palmer measure (Line 7 10).

\footnotetext{
${ }^{14}$ http: / / www . w3 .org/Mobile/CCPP/

${ }^{15}$ http: / / www. cen-ltso.net/main.aspx?put $=230$
} 
The communities with the highest values of similarity between the user's interests and community's interests are selected (Line10).

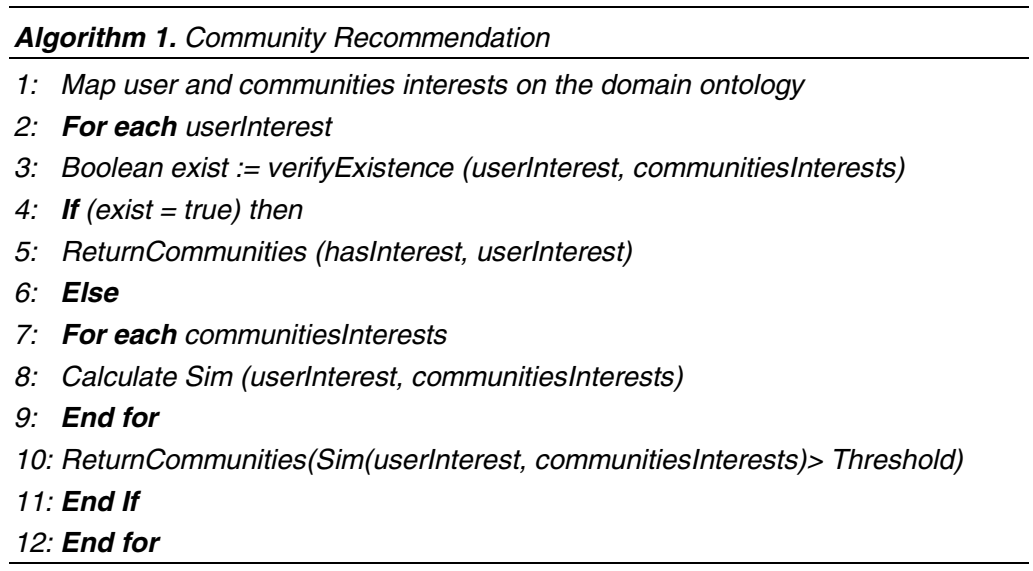

\subsubsection{Community Search}

With Taldea, the user can enter queries in natural language to search for communities. The query will be annotated through TextAnnot ${ }^{9}$. Using the results of this annotation, a Sparql query is formulated with the concepts results in the clause Where.

Table 3. Example of user query

I'm looking for orchid community

Table 4. Example of query formulation

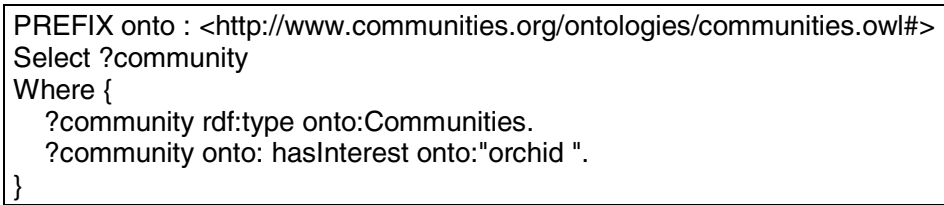

The two concepts 'orchid' and 'community', result of the annotation process, are used to build the clause Where of the Sparql query as shown in Table 4.

This module of the application allows users to query the community knowledge base without using a complex query language.

\section{Conclusion}

Taldea is a community's application that helps users access to communities and organize social exchanges between users in a geographic territory. In this paper, we present the first module of Taldea allowing user create or find a community. Taldea 
offers three ways to access a community; recommendation, search and creation of a community. On the one hand, the ontological modeling community adds a semantic layer to information and facilitates the user reach to communities and information retrieval within the community. On the other hand, it infers automatically new knowledge for further enrichment of community description. The use of standards like FOAF for describing the user's profile ensures accessibility and interoperability of data within communities.

Our immediate plan is to enrich the description of the community to include a spatio-temporal contextualization of social exchanges between users. In future work, several issues will be investigated. We plan to include a description of services provided for each kind of community and deploy Taldea in the software platform Kalimucho for deploying reconfigurable distributed applications.

\section{References}

1. Deparis, E., Abel, M.H., Mattioli, J.: Modeling a social collaborative platform with standard ontologies. In: International Workshop on Knowledge Acquisition, Reuse and Evaluation, Dijon, France, pp. 167-173 (2011)

2. Leprovost, D., Abrouk-Gouaich, L., Gross-Amblard, D.: Discovering implicit communities in web forums through ontologies. International Journal of Web Intelligence and Agent Systems (2012)

3. Ma, Y., Audibert, L., Nazarenko, A.: Formal Description of Resources for Ontology-based Semantic Annotation. In: The Seventh International Conference on Language Resources and Evaluation (2010)

4. Raad, E., Chbeir, R., Dipanda, A.: User Profile Matching in Social Networks. In: International Conference on Network-Based Information Systems (2010)

5. Sathik, M., Senthamarai, K., Rasheed, A.: Comparative Analysis of Community Discovery Methods in Social Networks. International Journal of Computer Applications 14(8) (2011)

6. Slimani, T., Ben Yaghlane, B., Mellouli, K.: A new similarity measure based on edge counting. In: Proceedings of the Seventeenth International Waset Conference, vol. 17 (2006)

7. Vercoutier, L., Maret, P.: Introducing Web Intelligence for communities. Web Intelligence and Agent Systems Journal (WIAS) 10(1), 89-92 (2012)

8. Weiser, M.: The Computer for the 21st Century. Scientific American Special Issue on Communications, Computers, and Networks (1991)

9. Wu, Z., Palmer, M.: Verb semantics and lexical selection. In: Proceedings of the 32nd Annual Meeting of the Associations for Computational Linguistics, pp. 133-138 (1994) 